\title{
A Comparison of Oral Gabapentin and Oral Pregabalin as Preemp- tive Analgesia for Orthopedic Surgery Under Spinal Anaesthesia
}

\author{
Jain Neena ${ }^{1}$, Bankapur Rahul' ${ }^{2}$ Lamba Preeti ${ }^{3}$, Saurav Singh ${ }^{4}$
}

\section{Senior Professor, Department of Anesthesiology, JLN Medical College, Ajmer, Rajasthan}

2. Senior Resident,Department of Anesthesiology, Karnataka Institute of Medical Sciences,Hubli, Karnataka- 580032

3. Senior Resident ,Department of Cardiac Anesthesia, Jawaharlal Nehru Medical College, Belgaum, Karnataka

4. Attending Consultant,Department of Neuroanaestheia, Max Super Speciality Hospital, Patparganj, Delhi

Corresponding author:

Dr. Rahul Bankapur, Department of Anesthesiology, Karnataka Institute of Medical Sciences,Hubli, Karnataka- 580032 Email id: rahul.bankapur@yahoo.com Ph no.-9008962859

Date of receiving: 15/2/2021

Date of review: 17/3/2021

Date of acceptance: 22/4/2021

\section{DOI:10.47799/pimr.0901.15}

\section{ABSTRACT}

Background and Aims: Gabapentin and pregabalin, by decreasing noxious stimulus induced excitatory neurotransmitter release at central nervous system, may attenuate central sensitization and eventually decrease development of postoperative pain. We evaluated preemptive analgesic efficacy of single dose of oral gabapentin $600 \mathrm{mg}$ and pregabalin $75 \mathrm{mg}$ for postoperative pain in patients undergoing lower limb orthopedic surgery under spinal anesthesia.

Material and methods: A prospective, randomized, double blind study was conducted on 70 patients aged between 18 to 60 years with ASA grade 1 and 2 posted for lower limb surgeries under spinal anaesthesia. Patients were allocated into Group A and Group B receiving oral gabapentin(600mg) and oral pregabalin $(75 \mathrm{mg}$ ) respectively 1.5 hours before surgery. Primary objective was assessing duration and quality of analgesia by Visual Analogue Scale (VAS) score at $2,4,6,8,10,12,16,20$ and 24 hours.Secondary objective was to assess total dose of rescue analgesic in first 24 hours, perioperative hemodynamic change and various side effects.

Statistical Analysis used: Categorical data was compared using Chi- square test. Quantitative parametric data was analysed using unpaired student t-test. $P$ value $<0.05$ was considered statistically significant.

Results: Mean duration of analgesia in Group A (10.53 \pm 2.686 hours) was longer than Group B $(7.943 \pm 3.199 \mathrm{hr})(\mathrm{P}=$ 0.0006). Mean number of analgesic dosesrequired in first 24 hourswere less in Group A (1.429 \pm 0.5021$)$ ascompared to
Group B (1.771 \pm 0.6897$)(P=0.0202)$.All patients remained hemodynamically stable with no significant side effects noted in either group.

Conclusion: We conclude that preemptive analgesic efficacy of oral gabapentin $600 \mathrm{mg}$ is better in comparison to oral pregabalin $75 \mathrm{mg}$ for patients posted for lower limb orthopedic surgeries under spinal anesthesia.

Keywords: gabapentin, pregabalin, preemptive analgesia, central sensitization, visual analogue scale.

\section{Original Article}

Introduction

The myelinated $\mathrm{Ad}$ and unmyelinated $\mathrm{C}$ nociceptors respond to strong painful stimuli and convert the chemical energy to electrical activity, which are conducted to the dorsal horn of CNS. From here, pain signals are transmitted to secondary nociceptive neurons known as specific neurons and wide-dynamic range neurons.The chemical mediators of pain pathway lead to peripheral sensitization of the nociceptors aiding to pain memory ${ }^{[1,2]}$

Preemptive analgesia, a preventive or pre incisional analgesia is an antinociceptive treatment, that prevents central hyperexcitability, sensitization, neuroplasticity evoked by the incisional and inflammatory injuries occurring during surgery and early postoperative period via altering the afferent input involved in pain.[3]Number of drugs are being used alone or as multi modal drug therapy for preemptive analgesia. ${ }^{[1]}$ 
Advantages of preemptive analgesia given along with neuraxial blocks include:prolonged duration of analgesia, decrease analgesic consumption and associated side effects, decrease in the development of central sensitization to pain, and prolong desensitization to pain postoperatively thus preventing development of chronic pain.

Gabapentin, structural analogue of gamma amino butyric acid, introduced in 1994 is a novel antiepileptic agent used basically in refractory partial seizures and many pain syndromes. It has been recommended routinely in pain management nowadays. ${ }^{[4,5]}$ Pregabalin introduced in 2004, is a newer structural analogue of gamma aminobutyric acid substituted at the 3-position; which facilitates diffusion across the blood-brain barrier. ${ }^{[6,7]}$ Pregablin is found to be superior in pharmacokinetic profile which is six times more potent than gabapentin. Pregabalin has much higher bioavailability $(90 \%$ vs. 33-66\%) with low intersubject variability. ${ }^{[6]}$

Mechanism of action of gabapentin and pregabalin, most likely involves inhibition of neuronal a2d subunit voltagegated calcium currents (VGCC) and suppression of excitatory neurotransmitter release like glutamate leading to decreased postsynaptic excitatory responses. These drugs thus act by inhibiting the neuro modulatory pain pathways that are responsible for development of acute and acute-chronic pain postoperatively. ${ }^{[6,7]}$ Both the drugs have unique and good pharmacokinetic profile. Side effects like nausea, vomiting, dizziness, sedation and visual disturbances seen with higher doses has limited their use in daily practice. ${ }^{[8-10]}$

Both gabapentin and pregabalin have been used in postoperative pain control but very few studies have compared the analgesic efficacy. Therefore, we evaluated pre-emptive analgesic efficacy of single dose of oral gabapentin $600 \mathrm{mg}$ and pregabalin $75 \mathrm{mg}$ for postoperative pain in patients undergoing lower limb orthopedic surgery under spinal anaesthesia.

\section{Materials and Methods:}

- A prospective, randomized, double blind clinical study was initiated after clearance from institutional ethical committee.(IEC/ 268/Educational -III/MCA/2018 Dated 06-022018 (Jawaharlal Nehru Medical College, Ajmer, Rajasthan and clinical trial registry number CTRI/2019/01/017050)

Sample size was calculated to be 35 patients to be randomly allocated in each group keeping a power of 0.8 , a error of 0.05 and allowing for study error and attrition (Figure 1).

After counselling regarding the study, written and informed consent was obtained. A total of 70 adult patients (18 - 60 years) with American Society of Anaesthesiologists (ASA) physical status 1 and 2, posted for elective lower limb orthopaedic surgery were allocated into two groups using computer generated table of random numbers, withGroup $A$ receiving oral gabapentin $600 \mathrm{mg}$ and Group B receiving oral pregabalin $1.5 \mathrm{hr}$ before surgery.

All patients included in the study were kept nil per oral for 8 hrs. [11]Routine vitals monitoring was done perioperatively. Patients were preloaded with $10 \mathrm{ml} / \mathrm{kg}$ ringer lactate followed by spinal anaesthesia using $15 \mathrm{mg}$ of $0.5 \%$ bupivacaine heavy. After surgery patients were transferred to post-operative ward.

Duration of analgesia was recorded from loss of pin prick sensation at T12 - L1to when patient demanded for rescue analgesia or VAS $>3$. Post-operative VAS score was monitored at $2,4,6,8,10,12,16,20,24$ hours. [4]Pain was assessed perioperatively using standard $10 \mathrm{~cm}$ visual analogscale with 0 corresponding to no pain and 10 worst possible pain. [12]

Total number of rescue analgesic (diclofenac $75 \mathrm{mg}$ intramuscular ) doses, hemodynamic parameters, sedation (assessed using Ramsey Sedation Scale), side effects like nausea, vomiting and dizziness were recorded both intra and postoperatively for first 24 hours. [13-15]

Variables were analysed statistically and expressed as mean \pm standard deviation. Categorical data was compared using Chi- square test. Quantitative parametric data was analysed using unpaired student t-test. $P$ value $<0.05$ was considered statistically significant. GraphPad Prism software version 6.01 for Windows (GraphPad Software Inc., La Jolla California, USA, 2012) was used for data tabulation and analysis.

Results :

Both groups were comparable in terms of demographic profile such age, sex, height, weight, ASA physical status, site of surgery with no statistically significant difference between two groups.

Duration of analgesia was longer in Group $A$ $(10.46 \pm 2.661 \mathrm{hr})$ than Group B $(7.943 \pm 3.199 \mathrm{hr})(\mathrm{P}$ value $=$ $0.0006)$ as shown in figure 2. Mean number of analgesic doses required in first 24 hours were less in Group $A(1.429 \pm 0.5021)$ as compared to Group B $(1.771 \pm 0.6897)(P=0.0202)$ as shown in figure 3.

Mean VAS Score were noted at 2, 4,6,8,10,12,16,20,24 hour postoperatively in either group. The results were statistically significant at 4, 6,10,16,24 hours between two groups as shown in figure 4.

All the patients remained hemodynamically stable throughout the period of study. There was no statistically significant difference between the groups. Side effects like hypotension $(P$ value $=0.6903)$, nausea, dizziness $(P$ value $=$ 0.3138 )were comparable and all patient had Ramsey sedation score of 2. No episode of vomiting was recorded in either group. 


\section{Figures:}

\section{Figure 1: Consort Diagram}

Figure 3: Mean number of analgesic doses required in first 24 hours postoperatively.

Figure 4: Mean VAS score at various time interval in first 24 hour.

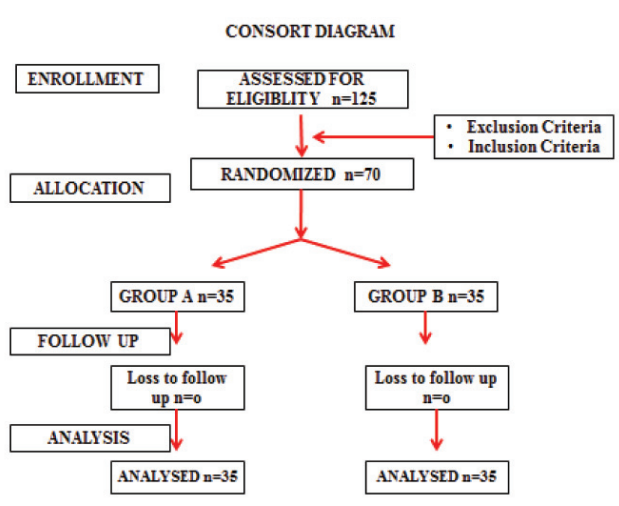

Figure 2-1

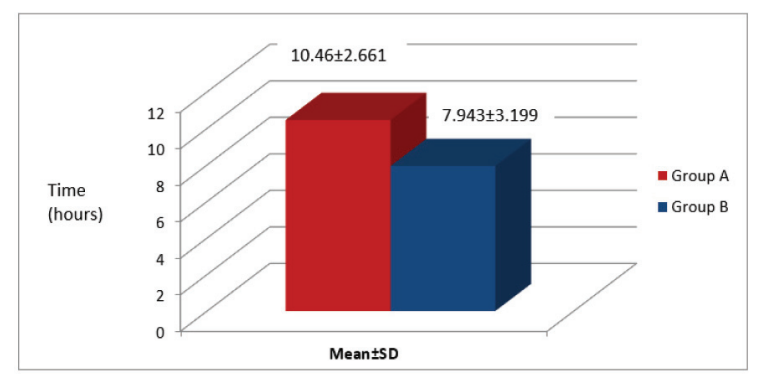

\section{Figure 3-1}

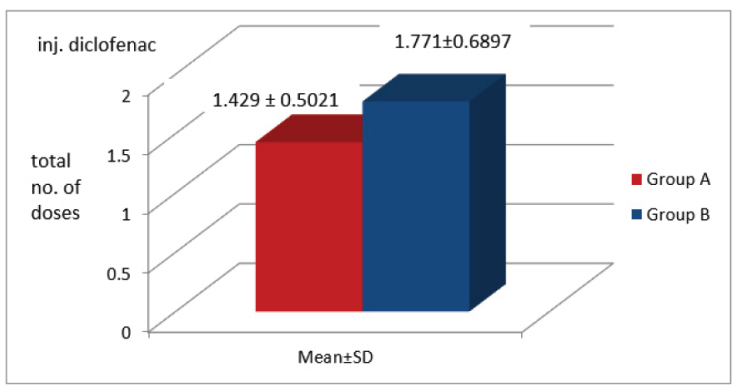

Figure 4-1

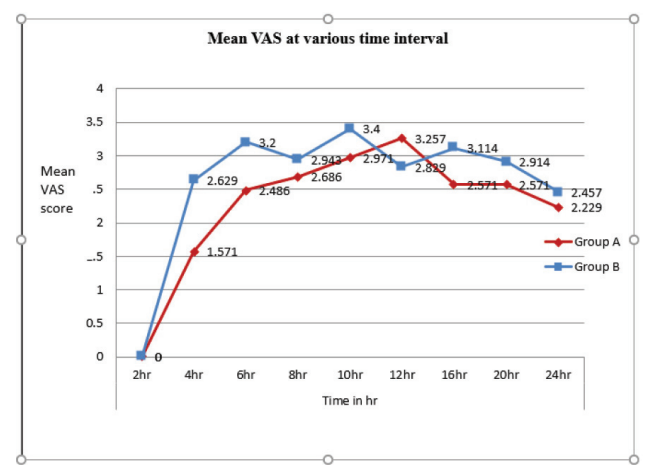

\section{Discussion:}

Acute pain left untreated can lead to long-term emotional and psychological distress and has the potential to develop into a chronic pain state which is much more difficult to manage. [1] Lower limb orthopaedic surgeries are associated with either neuropathic or inflammatory pain which may end up as chronic pain if not intervened at proper time. ${ }^{[5]}$

Opioids, nonsteroidal anti-inflammatory drugs (NSAID), NMDA antagonist, alpha 2 agonist are used as an adjuvant to epidural and spinal blocks as they have their own disadvantages. ${ }^{[16]}$ Anticonvulsants namely, gabapentin or pregabalin were used for preemptive analgesia in the present study as these drugs have minimal drug interaction and have good analgesic efficacy even with single dose. Moreover, they provide longer duration of post-operative analgesia compared to intrathecal bupivacaine alone. ${ }^{[17-20]}$ Previous studies which used higher doses had side effects which limited the use of these drugs routinely. Therefore, with paucity of literature for use of low doses, we compared pre-emptive analgesic efficacy of gabapentin $600 \mathrm{mg}$ with pregabalin $75 \mathrm{mg}$ in lower limb surgeries under spinal anesthesia.

Induja Rajendran et al , V Saraswati et al used higher doses of these drugs compared to our study with pregabalin proving to be superior but with most of the patients complaining of nausea, vomiting, dizziness, somnolence etc. ${ }^{[4,15]}$ Usha Bafna et al found pregabalin $150 \mathrm{mg}$ had a longer duration of analgesia in comparison to gabapentin $600 \mathrm{mg}$ (same dose as used in present study) with fewer side effect. ${ }^{[7]}$ Geetha Chamanalli et al compared two doses of pregabalin [75mg versus $150 \mathrm{mg}$ ] and found longer duration of analgesia in $150 \mathrm{mg}$ group. With minimal side effects in $75 \mathrm{mg}$ group. ${ }^{[17]}$ Freedman et al and Peng et al who used lower doses of pregabalin also found opioid sparing effect, lesser VAS score postoperatively, with reduced incidence of nausea compared to placebo. ${ }^{[21,22]} \mathrm{Hence}$, with optimal analgesic doses of gabapentin and pregabalin an attempt was made through our study to provide patient anxiolysis and reduce the incidence of nausea and vomiting along with prolonging postoperative analgesia.

Duration of analgesia was longer with gabapentin ( $P$ $=0.0006$ ) and had lesser VAS score in comparison to pregabalin throughout the period of study. Studies which used higher or similar doses compared to our study found significantly lower VAS score for gabapentin or pregabalin group in comparison to placebo group. ${ }^{[7,13,15,17]}$ Hence, with optimal dosing VAS score were lowered for painless recovery postoperatively. As fewer number of studies have noted mean VAS score at different interval, there is scope for future studies to note and confirm the same. 
Rescue analgesic dose required in first 24 hourswere lower with gabapentin ( $\mathrm{P}=0.0202)$, a finding which correlated with similar studies ${ }^{[4,6,13]}$ There was no need of opioids in either group and this was similar to observation made by Pragati Arora et al and Geetha chamanalli et al where injection diclofenac was sufficient to suffice the pain. ${ }^{[8,17]}$

Limitations of our study were, assessment of visual analog scale score is subjective, single dose of gabapentin and pregabalin used does not infer the long-term benefits to the patient. Control/placebo group has not been added to the study as both drugs have proved to increase post-operative analgesia and reduce analgesic requirement in most of the studies.

\section{Conclusion:}

Single oral dose of gabapentin $600 \mathrm{mg}$ given prior to spinal block in patient undergoing lower limb orthopedic surgeries provides longer duration of post-operative analgesia than single oral dose of pregabalin $75 \mathrm{mg}$ with minimum side effects. We recommend more use of concept of pre-emptive analgesia as a part of multimodal analgesia in day to day practice.

\section{REFERENCES}

1. Mishra AK, Afzal M, Mookerjee SS, Bandyopadhyay KH, Paul A. Pre-emptive analgesia : Recent trends and evidences. Indian J Pain. 2013; 27:114-20.

2. Dermot J. Kelly, Mahmood Ahmad MD, Sorin J. Brull. Preemptive analgesia I: physiological pathways and pharmacological modalities. CAN J ANESTH . 2001 July 11;48(10):1000-10.

3. Anil Verma, Sangeeta Arya , Sandeep Sahu, Indu Lata, H D Pandey, Harpreet Singh. To Evaluate the Role of Gabapentin as Preemptive Analgesic in Patients Undergoing Total Abdominal Hysterectomy in Epidural Anesthesia. Indian Journal of Anesthesia . 2008; 52 (4):428-431.

4. V Saraswat, Vishal Arora .Preemptive Gabapentin vs Pregabalin for Acute Postoperative Pain after Surgery under Spinal Anesthesia. Indian Journal of Anesthesia. 2008; 52 (6):829-834.

5. Mario Dauri, SkerdilajdFaria, Antonello Gatti, LudovicaCelidonio, Roberta Carpenedo, Alessandro F Sabato. Gabapentin and Pregabalin for the Acute Postoperative Pain Management. A Systematic-narrative Review of the Recent Clinical Evidences. Current Drug Targets.2009;10(8):716-733.

6. Ghai A, Gupta M, Hooda S, Singla D, Wadhera R. A randomized controlled trial to compare pregabalin with gabapentin for postoperative pain in abdominal hysterectomy.Saudi J Anaesth. 2011 july ;5(3):252-7.
7. Bafna U, Rajarajeshwaran K, Khandelwal M, Verma AP. A comparison of effect of preemptive use of oral gabapentin and pregabalin for acute post-operative pain after surgery under spinal anesthesia. J Anaesthesiol Clin Pharmacol. 2014;30:373-7.

8. Pragati Arora Trivedi1, Malini Mehta, Jayant Trivedi. Pre Emptive Gabapentin Versus Pregabalin for post Operative Analgesia after Abdominal Hysterectomy under Spinal Anesthesia. Int J Res Med. 2015; 4(1): 53-58.

9. Abhishek Bansal, Anurag Tewari, Shuchita Garg, Alka Gupta. Pregabalin: Pharmacology and Use in Pain Management. J Anaesth Clin Pharmacol. 2009; 25(3): 321326.

10. Bon Sebastian, Anand TippannaTalikoti, Kiran Nelamangala, Dinesh Krishnamurthy. Effect of Oral Pregabalin as Preemptive Analgesic in Patients Undergoing Lower Limb Orthopedic Surgeries under Spinal Anesthesia. Journal of Clinical and Diagnostic Research. 2016 Jul;10(7):01-04.

11. Practice Guidelines for Preoperative Fasting and the Use of Pharmacologic Agents to Reduce the Risk of Pulmonary Aspiration: Application to Healthy Patients Undergoing Elective Procedures: An Updated Report by the American Society of Anesthesiologists Committee on Standards and Practice Parameters. Anesthesiology 2011;114(3):495511. doi: 10.1097/ALN.0b013e3181fcbfd9.

12. Chooi CS, White AM, Tan SG, Dowling K, Cyna AM. Pain vs comfort scales after Caesarean section: a randomized trial. Br J Anaesth2013;110:780-87.

13. Akhavanakbari G, Entezariasl M, Isazadehfar K, Mirzarahimi T. The effects of oral pregabalin on postoperative pain of lower limb orthopedic surgery: A doubleblind, placebo-controlled trial. Perspect Clin Res. 2013;4:165-8.

14. Mackey, D. C., Butterworth, J. F., Mikhail, M. S., Morgan, G. E., and Wasnick, J. D. (2013). Morgan and Mikhail's clinical anesthesiology (5th ed.). New York, N.Y.: McGrawHill Education LLC.

15. Rajendran I, Basavareddy A, Meher BR, Srinivasan S. Prospective, randomised, double blinded controlled trial of gabapentin and pregabalin as pre emptive analgesia in patients undergoing lower abdominal and limb surgery under spinal anesthesia. Indian J Pain. 2014;28:155-9.

16. Vidhi Mahendru, Anurag Tewari, Sunil Katyal, Anju Grewal, M Rupinder Singh, RoohiKatyal. A comparision of intrarthecal dexmedetomidine, clonidine and fentanyl as adjuvants to hyperbaric bupivacaine for lower limb surgery : A double blind controlled study. J Anaesthesiol Clin Pharmacol. OCT-DEC 2013;29(4):496-502. 
17. Geetha Chamanhalli Rajappa, Saurabh Vig, YatishBevanaguddaiah, Tejesh C Anadaswamy. Efficacy of Pregabalin as Premedication for Post-Operative Analgesia in Vaginal Hysterectomy. Anesth Pain Med. 2016 june; 6(3):e34591.

18. TülinAKARSU, HülyaTÜR, CihanBOLAT, Ismail ÖZKAYNAK. Comparison of Pre-Emptive Pregabalin with Placebo and Diclofenac Combination for Postoperative Analgesia and Cognitive Functions After Laparoscopic Cholecystectomy. TurkiyeKlinikleri J Med Sci 2012;32(4):963-70.

19. Mohammed H Saeed, Anna W Krikor, Zaineb A Yaquob, Mustafa W Yihya, Hashim S Khayat. Preoperative Gabapentin in Laparoscopic Cholecystectomy. Bas J Surg. 2013 March 19; 19(1): 24-29.

20. Joon Ho Kim, Min Young Seo, Sang Duk Hong, Jungbok Lee, Seung-Kyu Chung, Hyo Yeol Kim, Hun-Jong Dhong. The Efficacy of Preemptive Analgesia With Pregabalin in Septoplasty. Clin Exp Otorhinolaryngol. 2014 Jun; 7(2): 102-105.

21. Freedman BM, O'Hara E. Pregabalin has opioid-sparing effects following augmentation mammaplasty. Aesthetic Surg J 2008;28:421-4.

22. Peng PW, Li C, Farcas E, Haley A, Wong W, Bender J. Use of low-dose pregabalin in patients undergoing laparoscopic cholecystectomy. Br J Anaesth2010;105:15561.

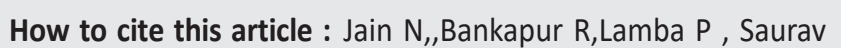
Singh . A Comparison of Oral Gabapentin and Oral Pregabalin as Preemptive Analgesia for Orthopedic Surgery Under Spinal Anaesthesia. Perspectives in Medical Research 2021; 9 (1):74-78 DOI:10.47799/pimr.0901.15

Sources of Support: Nil, Conflict of interest: None declared 